\title{
Incidence of G6PD deficiency among neonates with hyperbilirubinemia requiring phototherapy at postnatal wards of a tertiary care perinatal center, Dubai, UAE:A longitudinal cross-sectional study
}

\begin{abstract}
Purpose: To investigate the incidence of glucose 6 phosphate dehydrogenase (G6PD) deficiency in neonates with hyperbilirubinemia requiring phototherapy.

Methods: We conducted a longitudinal, cross-sectional, study that recruited all late preterm and term infants with hyperbilirubinemia who needed for phototherapy at postnatal wards of a tertiary care hospital in UAE through the period from April 2017 to January 2018. Neonates with an inborn error of metabolism, sepsis, or Neonatal intensive care unit (NICU) admission were excluded. A non-probability consecutive sampling technique was employed to recruit eligible neonates

Results: The present study included 658 late preterm and term infants with hyperbilirubinemia. The majority of the neonates were females $(51.4 \%)$ with a median (IQR) birth weight of 2846 (628) grams. Only 29 (4.4\%) neonates had a positive family history of G6PD deficiency. Sixty-nine (10.5\%) neonates had deficient G6PD activity. The association analysis showed that there were statistically significant associations between the presence of G6PD deficiency and the male gender $(\mathrm{p}<0.001)$, low birth weight $(\mathrm{p}$ $<0.001)$, and positive family history of G6PD deficiency $(\mathrm{p}<0.001)$

Conclusion: In conclusion, the present study showed that about $10 \%$ of the neonates admitted for phototherapy had G6PD deficiency in postnatal wards of a tertiary care hospital UAE. This finding highlights the critical role testing for G6PD to all newborns who are receiving phototherapy, especially those with unknown etiology or poor response to phototherapy. Further long-term studies are still needed to confirm our findings.
\end{abstract}

Volume 10 Issue 5 - 2020

\author{
Mahmoud Elhalik', Khaled El-Atawi², Swarup \\ Dash $^{3}$,Aida AbouNiaj ${ }^{4}$, Diana Dsouza 5 , Jemy \\ Jacob $^{6}$, Abrar Khan 7 , Rim Mahmouz 7 , Nancy \\ Augustin $^{8}$ \\ Consultant Neonatologist and Head of $\mathrm{NICU}, \mathrm{LWCH}$ \\ ${ }^{2}$ Consultant Neonatologist NICU, LWC \\ Senior Neonatologist, NICU, LWCH \\ ${ }^{4}$ Director of Nursing, $\mathrm{LWCH}$ \\ Nurse Supervisor, LWCH \\ ${ }^{6}$ Staff Nurse, LWCH \\ Specialist Neonatologist, NICU, LWCH \\ ${ }^{8}$ Charge Nurse, LWCH
}

Correspondence: Dr. Mahmoud Saleh Elhalik, Head of NICU, Latifa Women \& Children Hospital, DHA, Dubai, UAE, EmailmsEIHalik@dha.gov.ae

Received: September 21, 2020 | Published: October 26, 2020

Keywords: G6PD Deficiency; Hyperbilirubinemia; Phototherapy

\section{Introduction}

Glucose-6-phosphate dehydrogenase (G6PD) deficiency is one of the most prevalent genetic blood disorders which was estimated to affect up to 400 million people in $2009 .{ }^{1}$ The condition is characterized by an X-linked mutation in the structure of the G6PD enzyme, followed by a significant reduction in its activity and increase the liability of erythrocytes to damage by oxidative stress. ${ }^{2}$ Patients with G6PD are usually asymptomatic; however, they can present with sudden onset of acute hemolytic anemia after exposure to stress, oxidative substances such as fava beans, and certain medications like anti-malarial agents or sulfonamide antibiotic. ${ }^{3,4}$ The G6PD-related anemia can be complicated by neonatal jaundice and chronic nonspherocytic anemia. ${ }^{5,6}$ The association between neonatal jaundice and G6PD deficiency is of special concern as G6PD deficiency is a known risk factor for neonatal jaundice; in a recent meta-analysis, neonates with G6PD deficiency had three times the risk of hyperbilirubinemia and phototherapy than the ones with normal G6PD. ${ }^{7}$ Other reports showed that the prevalence of G6PD deficiency can be as high as $42 \%$ in neonates with hyperbilirubinemia. Despite this, it was reported that G6PD assessment is neglected in many centers treating neonates with hyperbilirubinemia. ${ }^{8,9}$

On the other hand, genetic disorders are common in Arab countries owing to the notably high consanguinity rates, advanced maternal age, large family size, and lack of effective perinatal counseling programs. ${ }^{10,11}$ The prevalence of G6PD deficiency in newborns was reported to range from 1-37\% across Arab countries making it ranked as one of the top five genetic diseases in the region. ${ }^{12,13}$ The situation is not different in the United Arab Emirates (UAE) with a reported prevalence of G6PD deficiency of 7.4\% ${ }^{14}$ In a recent pilot study from UAE, $30 \%$ of the neonatal jaundice cases had G6PD deficiency.

Latifa Women and Children's Hospital is a prominent tertiary care government hospital in the region, where approximately 4000 to 5000 births happen yearly. The normal care of the newborn requires an assessment of risk factors for the development of hyperbilirubinemia. Almost $100 \%$ of the newborns in the postnatal units have transcutaneous bilirubin and/or serum bilirubin done before they are discharged home. $20 \%$ of these babies are started on phototherapy in the postnatal units. In the present study, we investigated the incidence of G6PD deficiency in neonates with hyperbilirubinemia requiring phototherapy at postnatal units of Latifa Women and Children's Hospital, Dubai, UAE.

\section{Materials and methods}

We followed the STrengthening the Reporting of OBservational studies in Epidemiology (STROBE) statement recommendations during the preparation of this report. ${ }^{15}$ The study's protocol was approved by the local ethics and research committee of Latifa Women 
and Children Hospital. A written informed consent was obtained from parents of every eligible neonate prior to study enrollment.

\section{Study design, setting and participants}

We conducted a longitudinal, cross-sectional, study that recruited all late preterm and term infants with hyperbilirubinemia who were admitted for phototherapy at postnatal wards through the period from April 2017 to January 2018. Neonates with an inborn error of metabolism, sepsis, or Neonatal intensive care unit (NICU) admissions were excluded. A non-probability consecutive sampling technique was employed to recruit eligible neonates.

\section{Objectives}

\section{Primary objective:}

To detect incidence of G6PD deficiency among neonates (both late preterm and term) with hyperbilirubinemia requiring phototherapy and are admitted in postnatal ward of Latifa Women and Children's Hospital (LWCH), Dubai, UAE during the study period.

\section{Secondary objective:}

Various risk factors associated with G6PD deficiency (like gender, ethnicity, consanguinity, family history etc.).

To compare the course of Neonatal jaundice in G6PD deficient versus non deficient neonates.

\section{Data collection and study's visits}

The following data were collected: demographic characteristics of mothers, obstetric history, family history of G6PD deficiency, blood group of mother and baby, method of feeding, and the presence of G6PD deficiency. The quantitative assessment of G6PD activities was done according to hospital protocol. The primary outcome in the present study was the incidence of G6PD deficiency in neonates with hyperbilirubinemia requiring phototherapy at postnatal wards of Latifa Women and Children Hospital.

\section{Statistical analysis}

Data analysis was conducted using SPSS (Statistical Package for the Social Science; SPSS Inc., Chicago, IL, USA) version 22 for Microsoft Windows. The mean \pm standard deviation $( \pm \mathrm{SD})$ was used to express continuous data which are normally distributed and median $\&$ interquartile range, IQR) were used for non-parametric data; while frequencies with percentages were used to describe qualitative data. The unpaired Student's t-test and Chi-square test were used to assess the association between the presence of G6PD deficiency and characteristics of the newborns. A probability value (p-value) less than 0.05 was considered statistically significant.

\section{Results}

The present study included 658 late preterm and term infants with hyperbilirubinemia who needed phototherapy at postnatal wards through the period from April 2017 to January 2018. The median (IQR) maternal age was 31.4 (5.8) years old and gestational age was 37.6 (1.8) weeks. The majority of the neonates were females $(51.4 \%)$ with a median (IQR) birth weight of $2846 \pm 628$ gms. Only $29(4.4 \%)$ neonates had a positive family history of G6PD deficiency. Table 1 shows the characteristics of the included neonates. Sixty-nine (10.5\%) neonates had deficient G6PD activity (Figure 1). The association analysis showed that there were statistically significant associations between the presence of G6PD deficiency and the male gender ( $p$ $<0.001)$, low birth weight $(\mathrm{p}<0.001)$, and positive history of G6PD deficiency $(\mathrm{p}<0.001$; Table 2$)$.

Table I The characteristics of study participants

\begin{tabular}{ll}
\hline & N = 658 \\
\hline Maternal Age (years), Median (IQR) & $31.4(5.8)$ \\
Gestational Age (weeks), Median (IQR) & $37.6(1.8)$ \\
Male, no. (\%) & $320(48.6 \%)$ \\
Birth Weight (grams), Median (IQR) & $2846(628)$ \\
Emirates Nationally, No. (\%) & $387(58.8 \%)$ \\
Family history, No. (\%) & $29(4.4 \%)$ \\
Breastfeeding, No. (\%) & $186(25.5 \%)$ \\
\hline
\end{tabular}

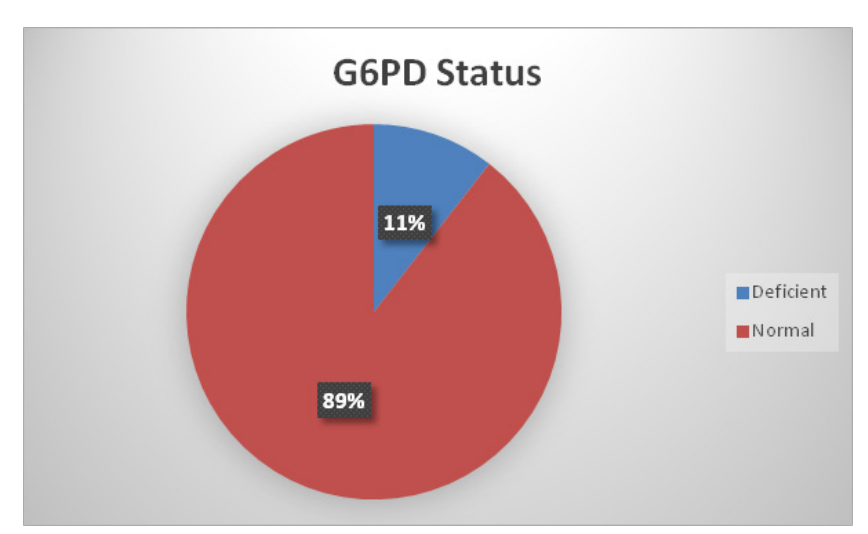

Figure I The incidence of G6PD Deficiency.

Table 2 The association between characteristics of study participants and G6PD status

\begin{tabular}{llll}
\hline & Deficient (N =69) & Normal (N =586) & P-value \\
\hline Maternal Age (years), Median (IQR) & $31.4(5.6)$ & $31.3(5.8)$ & 0.79 \\
Gestational Age (weeks), Median (IQR) & $38.1(1.5)$ & $37.5(1.9)$ & 0.18 \\
Male, no. (\%) & $56(81.2 \%)$ & $264(45.1 \%)$ & 0.001 \\
Birth Weight (Kg), Median (IQR) & $2747.1(892.4)$ & $2947.2(457.9)$ & 0.001 \\
Emirates Nationally, No. (\%) & $52(75.3)$ & $299(51)$ & 0.2 \\
Family history (G6PD deficiency), No. (\%) & $1 \mathrm{I}(15.9 \%)$ & $18(3.1 \%)$ & 0.001 \\
Breastfeeding, No. (\%) & $21(30.4 \%)$ & $165(28.2 \%)$ & 0.72 \\
\hline
\end{tabular}

Citation: Elhalik M, El-Atawi K, Dash S, et al. Incidence of G6PD deficiency among neonates with hyperbilirubinemia requiring phototherapy at postnatal wards of a tertiary care perinatal center, Dubai, UAE:A longitudinal cross-sectional study.J Pediatr Neonatal Care. 2020;10(5): I34-I37. 


\section{Discussion}

G6PD deficiency accounts for a considerable proportion of the cases with neonatal hyperbilirubinemia; however, many centers lack standardized protocols for the screening of G6PD activity, especially in neonates with no other identifiable cause of hyperbilirubinemia. In the present study, we found that $10.5 \%$ of the neonates admitted for phototherapy had G6PD deficiency. In addition, patients with G6PD deficiency were more likely to be males, have lower birth weight, and more likely to have a positive family history of G6PD deficiency.

Neonatal jaundice affects up to $85 \%$ of all live births and can cause significant morbidity and mortality to newborns. ${ }^{16}$ In its severe form, neonatal jaundice can lead to neurological complications such as impaired neurocognitive development, cerebral palsy, neuropathy, deafness, and, if left untreated, death. ${ }^{17,18}$ On the other hand, the G6PD enzyme is the catalyst of the first step in pentosephosphate pathway which ensures cellular protection against oxidative damage. ${ }^{19}$ Thus, G6PD deficiency can be associated with excessive damage of erythrocytes, acute hemolytic anemia, and indirect hyperbilirubinemia. ${ }^{6}$ In the preset study, we found that $10.5 \%$ of the neonates admitted for phototherapy had G6PD deficiency. In concordance with our findings, Dawodu et al. ${ }^{20}$ reported that, out of 85 neonates with hyperbilirubinemia at Al Ain Hospital of UAE, 9.5\% had G6PD deficiency. In a meta-analysis of five retrospective study, Liu et al. ${ }^{7}$ found that the prevalence of G6PD deficiency was $11.7 \%$ among neonates with hyperbilirubinemia. From Egypt, Abo El Fotoh and Rizk ${ }^{21}$ reported that the prevalence of G6PD in neonatal hyperbilirubinemia was $8.9 \%$. Notably, a recent report from Bahrain and Iran found that $42 \%$ and $18.1 \%$ of neonates with indirect hyperbilirubinemia had G6PD deficiency, respectively. ${ }^{22,23}$

Various risk factors were described in patients with G6PD deficiency such as male gender, African race, consanguinity, and positive family history. ${ }^{24}$ In the present study, we found that neonates with G6PD deficiency were more likely to be males, have lower birth weight, and more likely to have a positive family history of G6PD deficiency. In line with these findings, two reports from Yemen showed that G6PD deficiency was more prevalent in males and child of consanguinity marriage. ${ }^{23,25}$ In Isa's study, neonates with G6PD deficiency were more likely to be males. ${ }^{22}$

We acknowledge that the present study has a number of limitations. The study was conducted in one perinatal center only, which may affect the generalizability of our findings. Another limitation is the disproportional sample size to previous similar reports.

\section{Conclusion}

In conclusion, the present study showed that about $10 \%$ of the neonates admitted for phototherapy had G6PD deficiency in UAE. This finding highlights the critical role testing for G6PD to all newborns who are receiving phototherapy, especially those with unknown etiology or poor response to phototherapy. Further longterm studies are still needed to confirm our findings.

\section{Conflicts of interest}

All authors confirm no financial or personal relationship with a third party whose interests could be positively or negatively influenced by the article's content.

\section{Funding source}

None (authors confirm they did not receive any funding to do this work).

\section{References}

1. Nkhoma ET, Poole C, Vannappagari V, et al. The global prevalence of glucose-6-phosphate dehydrogenase deficiency: A systematic review and meta-analysis. Blood Cells Mol Dis. 2009;42(3):267-278.

2. Luzzatto L, Nannelli C, Notaro R. Glucose-6-Phosphate Dehydrogenase Deficiency. Hematol Oncol Clin North Am. 2016;30:373-393.

3. Iranpour R, Akbar MR, Haghshenas I. Glucose-6-Phosphate Dehydrogenase Deficiency in Neonates. Indian J Pediatr. 2003;70:855857.

4. Khan M. Glucose 6 phosphate dehydrogenase deficiency in adults. $J$ Coll Physicians Surg Pakistan. 2004;14:400-403.

5. Eberle SE, Rosolen NG, Urtasun C, et al. Glucose 6 phosphate dehydrogenase deficiency. A case series. Arch Argent Pediatr. 2011;109(4):354-357.

6. Monteiro WM, Franca GP, Melo GC, et al. Clinical complications of G6PD deficiency in Latin American and Caribbean populations: Systematic review and implications for malaria elimination programmes. Malar J. 2014;13

7. Liu H, Liu W, Tang X, et al. Association between G6PD Deficiency and Hyperbilirubinemia in Neonates: A Meta-Analysis. Pediatr Hemato Oncol. 2015;32(2):92-98.

8. Kaplan M, Herschel M, Hammerman C, et al. Hyperbilirubinemia among African American, glucose-6-phosphate dehydrogenase-deficient neonates. Pediatrics. 2004;114.

9. Kaplan M, Algur N, Hammerman C. Onset of jaundice in glucose-6-phosphate dehydrogenase - Deficient neonates. Pediatrics.2001;108(4):956-959.

10. Bayoumi RA, Yardumian A. Genetic disease in the Arab world. $\mathrm{Br} \mathrm{Med}$ J. 2006;333(7573):819.

11. Al-Gazali L, Hamamy H, Al-Arrayad S. Genetic disorders in the Arab world. BMJ. 2006;333(573):831-834.

12. Nkhoma ET, Poole C, Hall S, et al. Characterizing the Global Prevalence of Glucose-6-Phosphate Dehydrogenase Deficiency. Ann Epidemiol. 2007; $17: 747$.

13. Alharbi KK, Khan IA. Prevalence of glucose-6-phosphate dehydrogenase deficiency and the role of the A-variant in a Saudi population. J Int Med Res. 2014;42(5):1161-1167.

14. Amro SA Bin, Al Zaabi E, Hussain S, et al. Molecular characterization of glucose-6-phosphate dehydrogenase deficiency in Abu Dhabi District, United Arab Emirates. Trop J Pharm Res. 2014;13(5):731-737.

15. Elm E Von, Altman DG, Egger M, et al. The Strengthening the Reporting of Observational Studies in Epidemiology ( STROBE ) Statement : Guidelines for reporting observational studies. Int J Surg. 2014;12:1495-1499.

16. Bhutani VK, Zipursky A, Blencowe H, et al. Neonatal hyperbilirubinemia and rhesus disease of the newborn: Incidence and impairment estimates for 2010 at regional and global levels. Pediatr Res. 2013;74:86-100.

17. Mwaniki MK, Atieno M, Lawn JE, et al. Long-term neurodevelopmental outcomes after intrauterine and neonatal insults: A systematic review. Lancet. 2012;379(9814):445-452.

18. Slusher TM, Zamora TG, Appiah D, et al. Burden of severe neonatal jaundice: A systematic review and meta-analysis. BMJ Paediatr Open. $2017 ; 1$.

19. Luzzatto L. Glucose 6-phosphate dehydrogenase deficiency: From genotype to phenotype. Haematologica. 2006;91(10):1303-1306.

20. Dawodu A, Qureshi MM, Moustafa IA, et al. Epidemiology of clinical hyperbilirubinaemia in Al Ain, United Arab Emirates. Ann Trop Paediatr. 1998;18(2):93-99.

Citation: Elhalik M, El-Atawi K, Dash S, et al. Incidence of G6PD deficiency among neonates with hyperbilirubinemia requiring phototherapy at postnatal wards of a tertiary care perinatal center, Dubai, UAE:A longitudinal cross-sectional study.J Pediatr Neonatal Care. 2020; I 0(5): I34-I37. 
21. M Abo El Fotoh WM, Rizk MS. Prevalence of glucose-6-phosphate dehydrogenase deficiency in jaundiced Egyptian neonates. J Matern Neonatal Med. 2016;29(5):3834-3837.

22. Isa HM, Mohamed MS, Mohamed AM, et al. Neonatal indirect hyperbilirubinemia and glucose-6-phosphate dehydrogenase deficiency. Korean J Pediatr. 2017;60:106-111.

23. Pahlavanzadeh M, Hekmatimoghaddam S, Teremahi Ardestani M, et al. G6PD Enzyme Deficiency in Neonatal Pathologic Hyperbilirubinemia in Yazd. Iran J Pediatr Hematol Oncol. 2013;3(2):69-72.
24. Carvalho CG, Castro SM, Santin AP, et al. Glucose-6-phosphatedehydrogenase deficiency and its correlation with other risk factors in jaundiced newborns in Southern Brazil. Asian Pac J Trop Biomed. 2011;1(2):110-113.

25. Abdul-Ghani R, Mahdy MAK, Saif-Ali R, et al. Glucose-6-phosphate dehydrogenase deficiency among Yemeni children residing in malariaendemic areas of Hodeidah governorate and evaluation of a rapid diagnostic test for its detection. Malar J. 2016;15. 